\title{
Closed-Form Solution of a Rational Difference Equation
}

\author{
Tarek F. Ibrahim $\mathbb{D}^{1},{ }^{1}$ Abdul Qadeer Khan $\mathbb{D}^{2},{ }^{2}$ Burak Oğul $\mathbb{D},{ }^{3}$ and Dağistan Şimşek ${ }^{4}$ \\ ${ }^{1}$ Department of Mathematics, Faculty of Sciences, Mansoura University, Mansoura, Egypt \\ ${ }^{2}$ Department of Mathematics, University of Azad Jammu and Kashmir, Muzaffarabad 13100, Pakistan \\ ${ }^{3}$ Department of Management Information Systems, School of Applied Science, Istanbul Aydin University, Istanbul, Turkey \\ ${ }^{4}$ Department of Engineering Basic Sciences, Faculty of Engineering and Natural Sciences, Konya Technical University, \\ Konya, Turkey
}

Correspondence should be addressed to Abdul Qadeer Khan; abdulqadeerkhan1@gmail.com

Received 22 August 2021; Accepted 8 November 2021; Published 29 November 2021

Academic Editor: M Syed Ali

Copyright (C) 2021 Tarek F. Ibrahim et al. This is an open access article distributed under the Creative Commons Attribution License, which permits unrestricted use, distribution, and reproduction in any medium, provided the original work is properly cited.

In this paper, we study the solution of the difference equation $\Omega_{m+1}=\left(\Omega_{m-(7 q+6)} /\left(1+\prod_{t=0}^{5} \Omega_{m-(q+1) t-q}\right)\right)$, where the initials are positive real numbers.

\section{Introduction}

Difference equations appear naturally as discrete analogues in many sciences such as biology, ecology, and physics. In recent years, many authors studied the solution form of difference equations. For instance, Cinar [1-3] studied

$$
\begin{aligned}
& \Omega_{m+1}=\frac{\Omega_{m-1}}{1+a \Omega_{m} \Omega_{m-1}}, \quad m=0,1, \ldots, \\
& \Omega_{m+1}=\frac{\Omega_{m-1}}{-1+a \Omega_{m} \Omega_{m-1}}, \quad m=0,1, \ldots, \\
& \Omega_{m+1}=\frac{a \Omega_{m-1}}{1+b \Omega_{m} \Omega_{m-1}}, \quad m=0,1, \ldots,
\end{aligned}
$$

respectively.

DeVault et al. [4] examined

$$
\Omega_{m+1}=\frac{A}{\Omega_{m}}+\frac{1}{\Omega_{m-2}}, \quad m=0,1, \ldots .
$$

Elsayed [5] dealt with

$$
\Omega_{m+1}=a+\frac{b \Omega_{m-l}+c \Omega_{m-q}}{d \Omega_{m-l}+e \Omega_{m-q}}, \quad m=0,1, \ldots
$$

Simsek et al. [6-9] studied

$$
\begin{aligned}
& \Omega_{m+1}=\frac{\Omega_{m-3}}{1+\Omega_{m-1}}, \quad m=0,1, \ldots, \\
& \Omega_{m+1}=\frac{\Omega_{m-5}}{1+\Omega_{m-2}}, \quad m=0,1, \ldots, \\
& \Omega_{m+1}=\frac{\Omega_{m-5}}{1+\Omega_{m-1} \Omega_{m-3}}, \quad m=0,1, \ldots, \\
& \Omega_{m+1}=\frac{\Omega_{m-3}}{1+\Omega_{m} \Omega_{m-1} \Omega_{m-2}}, \quad m=0,1, \ldots,
\end{aligned}
$$

respectively. For some more results concerning difference equations, we refer the reader to [10-21].

In this work, we deal with the following nonlinear difference equation:

$$
\Omega_{m+1}=\frac{\Omega_{m-(7 q+6)}}{1+\prod_{t=0}^{5} \Omega_{m-(q+1) t-q}}, \quad m=0,1, \ldots,
$$

where investigated. 


\section{Main Results}

Let $\bar{\Omega}$ be the unique equilibrium of equation (5); then,

$$
\bar{\Omega}=\frac{\bar{\Omega}}{1+\bar{\Omega} \cdot \bar{\Omega} \cdot \bar{\Omega} \cdot \bar{\Omega} \cdot \bar{\Omega} \cdot \bar{\Omega}} \Rightarrow \bar{\Omega}+\bar{\Omega}^{7}=\bar{\Omega} \Rightarrow \bar{\Omega}^{7}=0 \Rightarrow \bar{\Omega}=0,
$$

so $\bar{\Omega}=0$ is obtained. For every $q \geq 0$ and $m>q$, notation $i=\overline{q, m}$ means $i=q, q+1, \ldots, m$.

Theorem 1. For (5), the following statements are true:

(a) The sequences $\quad\left\{\left(\Omega_{(7 q+7) n t-n(7 q+6)}\right\}\right.$, $\left\{\left(\Omega_{(7 q+7) n t-n(7 q+5)}\right\}, \ldots,\left\{\left(\Omega_{(7 q+7) n}\right\}\right.\right.$ are decreased, and $a_{1}, a_{2}, \ldots, a_{7 q+7} \geq 0$ exists such that

$$
\lim _{n \longrightarrow \infty} \Omega_{(7 q+7) n-(7 q+6)+t}=a_{1+t}, \quad t=\overline{0,7 q+6} .
$$

(b)

$$
\prod_{t=0}^{6} \lim _{n \longrightarrow \infty} \Omega_{(7 q+7) n-(q+1) t-q+i}=0, \quad i=\overline{0, q},
$$

or

$$
\prod_{t=0}^{6} a_{(q+1) t+i}=0, \quad i=\overline{1, q+1} .
$$

(c) If there exists $n_{0} \in \mathbb{N}$ such that

$$
\prod_{t=0}^{5} \Omega_{m-(q+1) t-q} \geq \Omega_{m+1} \prod_{t=0}^{4} \Omega_{m-(q+1) t-q}
$$

for all $n \geq n_{0}$, then

$$
\lim _{n \longrightarrow \infty} \Omega_{m}=0 .
$$

(d) We can generate the following formulas:

$$
\begin{aligned}
& \Omega_{(7 q+7) n+r(q+1)+s+1}=\Omega_{(r-7)(q+1)+s+1}(1- \\
& \frac{\left(\prod_{m=1}^{7} \Omega_{-(m q+m-1)+s}\right) / \Omega_{(r-7)(q+1)+s+1}}{1+\left(\prod_{m=1}^{6} \Omega_{-(m q+m-1)+s}\right)} \times \\
& \left.\sum_{h=0}^{n} \prod_{k=1}^{7 h+r} \frac{1}{\prod_{m=1}^{6} \Omega_{(q+1) t-(m q+m-1)+s}+1}\right) .
\end{aligned}
$$

(e) If $\Omega_{(7 q+7) n+(t-1) q+t} \longrightarrow a_{(t-1) q+t} \neq 0$, then $\Omega_{(7 q+7) n+6 q+7}$ $\longrightarrow 0$ as $n \longrightarrow \infty$.

If $\Omega_{(7 q+7) n+t q+t} \longrightarrow a_{t q+t} \neq 0$, then $\Omega_{(7 q+7) n+7 q+7} \longrightarrow 0$ as $n \longrightarrow \infty . t=\overline{1,6}$.

Proof

(a) Firstly, for all $n \in \mathbb{N}$, from (5), one gets $\Omega_{m+1}<\Omega_{m-(7 q+6)}$. So,

$\lim _{n \longrightarrow \infty} \Omega_{(7 q+7) n-(7 q+6)+t}=a_{1+t}, \quad t=\overline{0,7 q+6}$. (b) In view of equation (5),

$$
\Omega_{(7 q+7) n+1}=\frac{\Omega_{(7 q+7) n-(7 q+6)}}{1+\prod_{t=0}^{5} \Omega_{(7 q+7) n-(q+1) t-q}} .
$$

Then,

$$
\begin{aligned}
& \lim _{n \longrightarrow \infty} \Omega_{(7 q+7) n+1}=\lim _{n \longrightarrow \infty} \frac{\Omega_{(7 q+7) n-(7 q+6)}}{1+\prod_{t=0}^{5} \Omega_{(7 q+7) n-(q+1) t-q}}, \\
& \prod_{t=0}^{6} \lim _{n \longrightarrow \infty} \Omega_{(7 q+7) n-(q+1) t-q+i}=0, \quad i=\overline{0, q},
\end{aligned}
$$

or

$$
\prod_{t=0}^{6} a_{(q+1) t+i}=0, \quad i=\overline{1, q+1}
$$

is obtained.

(c) If there exists $n_{0} \in \mathbb{N}$ such that

$$
\prod_{t=0}^{5} \Omega_{m-(q+1) t-q} \geq \Omega_{m+1} \prod_{t=0}^{4} \Omega_{m-(q+1) t-q} \quad \text { for all } n \geq n_{0},
$$

then if $i=\overline{0, q}$,

$$
\begin{aligned}
a_{1+i} & \leq a_{q+2+i} \leq a_{2 q+3+i} \leq a_{3 q+4+i} \leq a_{4 q+5+i} \\
& \leq a_{5 q+6+i} \leq a_{6 q+7+i} \leq a_{1+i},
\end{aligned}
$$

and we obtained the result.

(d) Subtracting $\Omega_{m-(7 q+6)}$ from both sides in (5), we have

$$
\Omega_{m+1}-\Omega_{m-(7 q+6)}=\frac{1}{1+\prod_{t=0}^{5} \Omega_{m-(q+1) t-q}}\left(\Omega_{m-q}-\Omega_{m-(8 q+7)}\right),
$$

and the following formula, for $n \geq q+1$,

$$
\begin{aligned}
& \Omega_{(q+1) n-\left((q+1)^{2}-1\right)^{-}} \\
& \Omega_{(q+1) n-\left[(q+2)^{2}+(5 q+3)\right]} \\
& =\left(\Omega_{1}-\Omega_{-(7 q+6)}\right) \prod_{k=1}^{n-(q+1)} \frac{1}{1+\prod_{t=0}^{5} \Omega_{(q+1) k-(q+1) t-q}}, \\
& \vdots \\
& \Omega_{(q+1) n-\left((q+1)^{2}-(q+1)\right)}-\Omega_{(q+1) n-\left[(q+2)^{2}+(4 q+3)\right]}, \\
& =\left(\Omega_{q+1}-\Omega_{-(6 q+6)}\right) \prod_{k=1}^{n-(q+1)} \frac{1}{1+\prod_{t=0}^{5} \Omega_{(q+1) k-(q+1) t}} .
\end{aligned}
$$

From (20), we get 


$$
\begin{gathered}
\Omega_{(7 q+7) n+1}-\Omega_{-(7 q+6)}=\left(\Omega_{1}-\Omega_{-(7 q+6)}\right) \sum_{h=0}^{n} \prod_{k=1}^{7 h} \frac{1}{\prod_{t=0}^{5} \Omega_{(q+1) k-(q+1) t-q}+1}, \\
\cdot \\
\cdot \\
\Omega_{(7 q+7) n+q+1}-\Omega_{-(6 q+6)}=\left(\Omega_{q+1}-\Omega_{-(6 q+6)}\right) \sum_{h=0}^{n} \prod_{k=1}^{7 h} \frac{1}{\prod_{t=0}^{5} \Omega_{(q+1) k-(q+1) t}+1} .
\end{gathered}
$$

So,

$$
\begin{gathered}
\Omega_{(7 q+7) n+q+2}-\Omega_{-(6 q+5)}=\left(\Omega_{1}-\Omega_{-(7 q+6)}\right) \sum_{h=0}^{n} \prod_{k=1}^{7 h+1} \frac{1}{\prod_{t=0}^{5} \Omega_{(q+1) k-(q+1) t-q}+1}, \\
\cdot \\
\cdot \\
\Omega_{(7 q+7) n+2 q+2}-\Omega_{-(5 q+5)}=\left(\Omega_{q+1}-\Omega_{-(6 q+6)}\right) \sum_{h=0}^{n} \prod_{k=1}^{7 h+1} \frac{1}{\prod_{t=0}^{5} \Omega_{(q+1) k-(q+1) t}+1} .
\end{gathered}
$$

Also,

$$
\begin{gathered}
\Omega_{(7 q+7) n+2 q+3}-\Omega_{-(5 q+4)}=\left(\Omega_{1}-\Omega_{-(7 q+6)}\right) \sum_{h=0}^{n} \prod_{k=1}^{7 h+2} \frac{1}{\prod_{t=0}^{5} \Omega_{(q+1) k-(q+1) t-q}+1}, \\
\cdot \\
\cdot \\
\Omega_{(7 q+7) n+3 q+3}-\Omega_{-(4 q+4)}=\left(\Omega_{q+1}-\Omega_{-(6 q+6)}\right) \sum_{h=0}^{n} \prod_{k=1}^{7 h+2} \frac{1}{\prod_{t=0}^{5} \Omega_{(q+1) k-(q+1) t}+1} .
\end{gathered}
$$


Moreover,

$$
\begin{gathered}
\Omega_{(7 q+7) n+3 q+4}-\Omega_{-(4 q+3)}=\left(\Omega_{1}-\Omega_{-(7 q+6)}\right) \sum_{h=0}^{n} \prod_{k=1}^{7 h+3} \frac{1}{\prod_{t=0}^{5} \Omega_{(q+1) k-(q+1) t-q}+1}, \\
\cdot \cdot \\
\cdot \\
\Omega_{(7 q+7) n+4 q+4}-\Omega_{-(3 q+3)}=\left(\Omega_{q+1}-\Omega_{-(6 q+6)}\right) \sum_{h=0}^{n} \prod_{k=1}^{7 h+3} \frac{1}{\prod_{t=0}^{5} \Omega_{(q+1) k-(q+1) t}+1} .
\end{gathered}
$$

On the contrary,

$$
\begin{gathered}
\Omega_{(7 q+7) n+4 q+5}-\Omega_{-(3 q+2)}=\left(\Omega_{1}-\Omega_{-(7 q+6)}\right) \sum_{h=0}^{n} \prod_{k=1}^{7 h+4} \frac{1}{\prod_{t=0}^{5} \Omega_{(q+1) k-(q+1) t-q}+1}, \\
\cdot \cdot \\
\cdot \\
\Omega_{(7 q+7) n+5 q+5}-\Omega_{-(2 q+2)}=\left(\Omega_{q+1}-\Omega_{-(6 q+6)}\right) \sum_{h=0}^{n} \prod_{k=1}^{7 h+4} \frac{1}{\prod_{t=0}^{5} \Omega_{(q+1) k-(q+1) t}+1} .
\end{gathered}
$$

Also,

$$
\begin{gathered}
\Omega_{(7 q+7) n+5 q+6}-\Omega_{-(2 q+1)}=\left(\Omega_{1}-\Omega_{-(7 q+6)}\right) \sum_{h=0}^{n} \prod_{k=1}^{7 h+5} \frac{1}{\prod_{t=0}^{5} \Omega_{(q+1) k-(q+1) t-q}+1}, \\
\cdot \\
\cdot \\
\Omega_{(7 q+7) n+6 q+6}-\Omega_{-(q+1)}=\left(\Omega_{q+1}-\Omega_{-(6 q+6)}\right) \sum_{h=0}^{n} \prod_{k=1}^{7 h+5} \frac{1}{\prod_{t=0}^{5} \Omega_{(q+1) k-(q+1) t}+1} .
\end{gathered}
$$


Moreover,

$$
\begin{gathered}
\Omega_{(7 q+7) n+6 q+7}-\Omega_{-(q+)}=\left(\Omega_{1}-\Omega_{-(7 q+6)}\right) \sum_{h=0}^{n} \prod_{k=1}^{7 h+6} \frac{1}{\prod_{t=0}^{5} \Omega_{(q+1) k-(q+1) t-q}+1}, \\
\cdot \\
\cdot \\
\cdot \\
\Omega_{(7 q+7) n+7 q+7}-\Omega_{0}=\left(\Omega_{q+1}-\Omega_{-(6 q+6)}\right) \sum_{h=0}^{n} \prod_{k=1}^{7 h+6} \frac{1}{\prod_{t=0}^{5} \Omega_{(q+1) k-(q+1) t}+1} .
\end{gathered}
$$

Now, we get the above formulas:

$\Omega_{(7 q+7) n+r(q+1)+s+1}$

$=\Omega_{(r-7)(q+1)+s+1}$

$$
\begin{aligned}
& \cdot\left(1-\frac{\prod_{m=1}^{7}\left(\Omega_{-(m q+m-1)+s} / \Omega_{(r-7)(q+1)+s+1}\right)}{1+\left(\prod_{m=1}^{6} \Omega_{-(m q+m-1)+s}\right)}\right. \\
& \left.\times \sum_{h=0}^{n} \prod_{k=1}^{7 h+r} \frac{1}{\prod_{m=1}^{6} \Omega_{(q+1) t-(m q+m-1)+s}+1}\right),
\end{aligned}
$$

where $r=\overline{0,6}$ and $s=\overline{0, q}$ hold.

(e) Suppose that $a_{1}=a_{q+2}=a_{2 q+3}=a_{3 q+} 4=a_{4 q+5}=$ $a_{5 q+6}=a_{6 q+7}=0$. By $(\mathrm{d})$, we produce the following formulas:

$$
\begin{aligned}
\lim _{n \longrightarrow \infty} \Omega_{(7 q+7) n+1}= & \lim _{n \longrightarrow \infty} \Omega_{-(7 q+6)}\left(1-\frac{\prod_{t=0}^{5} \Omega_{-(q+1) t-q}}{\prod_{t=0}^{5} \Omega_{-(q+1) t-q}+1}\right. \\
& \left.\times \sum_{h=0}^{n} \prod_{k=1}^{7 h} \frac{1}{\prod_{t=0}^{5} \Omega_{-(q+1) k-(q+1) t-q}+1}\right), \\
a_{1}= & \Omega_{-(7 q+6)}\left(1-\frac{\prod_{t=0}^{5} \Omega_{-(q+1) t-q} \Omega_{-11+q}}{\prod_{t=0}^{5} \Omega_{-(q+1) t-q}+1} \sum_{h=0}^{\infty} \prod_{k=1}^{7 h} \frac{1}{\prod_{t=0}^{5} \Omega_{-(q+1) k-(q+1) t-q}+1}\right), \\
a_{1}= & 0 \Rightarrow \frac{\prod_{t=0}^{5} \Omega_{-(q+1) t-q}+1}{\prod_{t=0}^{5} \Omega_{-(q+1) t-q}}=\sum_{h=0}^{\infty} \prod_{k=1}^{7 h} \frac{1}{\prod_{t=0}^{5} \Omega_{(q+1) k-(q+1) t-q}+1} .
\end{aligned}
$$


Similarly,

$$
\begin{aligned}
\lim _{n \longrightarrow \infty} \Omega_{(7 q+7) n+q+2}= & \lim _{n \longrightarrow \infty} \Omega_{-(6 q+5)}\left(1-\frac{\prod_{t=0}^{6}\left(\Omega_{-(q+1) t-q} / \Omega_{-(6 q+5)}\right)}{\prod_{t=0}^{5} \Omega_{-(q+1) t-q}+1}\right. \\
& \left.\times \sum_{h=0}^{n} \prod_{k=1}^{7 h+1} \frac{1}{\prod_{t=0}^{5} \Omega_{-(q+1) k-(q+1) t-q}+1}\right) \\
a_{q+2}= & \Omega_{-(6 q+5)}\left(1-\frac{\prod_{t=0}^{6}\left(\Omega_{-(q+1) t-q} / \Omega_{-(6 q+5)}\right)}{\prod_{t=0}^{5} \Omega_{-(q+1) t-q}+1}\right. \\
& \left.\times \sum_{h=0}^{\infty} \prod_{k=1}^{7 h+1} \frac{1}{\prod_{t=0}^{5} \Omega_{-(q+1) k-(q+1) t-q}+1}\right) \\
a_{q+2}= & 0 \Rightarrow \frac{\prod_{t=0}^{5} \Omega_{-(q+1) t-q}+1}{\prod_{t=0}^{6}\left(\Omega_{-(q+1) t-q} / \Omega_{-(6 q+5)}\right.}=\sum_{h=0}^{\infty} \prod_{k=1}^{7 h+1} \frac{1}{\prod_{t=0}^{5} \Omega_{(q+1) k-(q+1) t-q}+1}
\end{aligned}
$$

Similarly,

$$
\begin{aligned}
\lim _{n \longrightarrow \infty} \Omega_{(7 q+7) n+2 q+3}= & \lim _{n \longrightarrow \infty} \Omega_{-(5 q+4)}\left(1-\frac{\prod_{t=0}^{6}\left(\Omega_{-(q+1) t-q} / \Omega_{-(5 q+4)}\right)}{\prod_{t=0}^{5} \Omega_{-(q+1) t-q}+1}\right. \\
& \left.\times \sum_{h=0}^{n} \prod_{k=1}^{7 h+2} \frac{1}{\prod_{t=0}^{5} \Omega_{-(q+1) k-(q+1) t-q}+1}\right), \\
a_{2 q+3}= & \Omega_{-(5 q+4)}\left(1-\frac{\prod_{t=0}^{6}\left(\Omega_{-(q+1) t-q} / \Omega_{-(5 q+4)}\right)}{\prod_{t=0}^{5} \Omega_{-(q+1) t-q}+1}\right. \\
& \left.\times \sum_{h=0}^{n} \prod_{k=1}^{7 h+2} \frac{1}{\prod_{t=0}^{5} \Omega_{-(q+1) k-(q+1) t-q}+1}\right), \\
a_{2 q+3}= & 0 \frac{\prod_{t=0}^{5} \Omega_{-(q+1) t-q}+1}{\prod_{t=0}^{6}\left(\Omega_{-(q+1) t-q} / \Omega_{-(5 q+4)}\right)}=\sum_{h=0}^{\infty} \prod_{k=1}^{7 h+2} \frac{1}{\prod_{t=0}^{5} \Omega_{(q+1) k-(q+1) t-q}+1} .
\end{aligned}
$$


Similarly,

$$
\begin{aligned}
\lim _{n \longrightarrow \infty} \Omega_{(7 q+7) n+3 q+4}= & \lim _{n \longrightarrow \infty} \Omega_{-(4 q+3)}\left(1-\frac{\prod_{t=0}^{6}\left(\Omega_{-(q+1) t-q} / \Omega_{-(4 q+3)}\right)}{\prod_{t=0}^{5} \Omega_{-(q+1) t-q}+1}\right. \\
& \left.\times \sum_{h=0}^{n} \prod_{k=1}^{7 h+3} \frac{1}{\prod_{t=0}^{5} \Omega_{-(q+1) k-(q+1) t-q}+1}\right), \\
a_{3 q+4}= & \Omega_{-(4 q+3)}\left(1-\frac{\prod_{t=0}^{6}\left(\Omega_{-(q+1) t-q} / \Omega_{-(4 q+3)}\right)}{\prod_{t=0}^{5} \Omega_{-(q+1) t-q}+1}\right. \\
& \left.\times \sum_{h=0}^{n} \prod_{k=1}^{7 h+3} \frac{1}{\prod_{t=0}^{5} \Omega_{-(q+1) k-(q+1) t-q}+1}\right) \\
a_{3 q+4}= & 0 \Rightarrow \frac{\prod_{t=0}^{5} \Omega_{-(q+1) t-q}+1}{\prod_{t=0}^{6}\left(\Omega_{-(q+1) t-q} / \Omega_{-(4 q+3)}\right)}=\sum_{h=0}^{\infty} \prod_{k=1}^{7 h+3} \frac{1}{\prod_{t=0}^{5} \Omega_{(q+1) k-(q+1) t-q}+1} .
\end{aligned}
$$

Similarly,

$$
\begin{aligned}
\lim _{n \longrightarrow \infty} \Omega_{(7 q+7) n+4 q+5}= & \lim _{n \longrightarrow \infty} \Omega_{-(3 q+2)}\left(1-\frac{\prod_{t=0}^{6}\left(\Omega_{-(q+1) t-q} / \Omega_{-(3 q+2)}\right)}{\prod_{t=0}^{5} \Omega_{-(q+1) t-q}+1}\right. \\
& \left.\times \sum_{h=0}^{n} \prod_{k=1}^{7 h+4} \frac{1}{\prod_{t=0}^{5} \Omega_{-(q+1) k-(q+1) t-q}+1}\right), \\
a_{4 q+5}= & \Omega_{-(3 q+2)}\left(1-\frac{\prod_{t=0}^{6}\left(\Omega_{-(q+1) t-q} / \Omega_{-(3 q+2)}\right)}{\prod_{t=0}^{5} \Omega_{-(q+1) t-q}+1}\right. \\
& \left.\times \sum_{h=0}^{n} \prod_{k=1}^{7 h+4} \frac{1}{\prod_{t=0}^{5} \Omega_{-(q+1) k-(q+1) t-q}+1}\right) \\
a_{4 q+5}= & 0 \Rightarrow \frac{\prod_{t=0}^{5} \Omega_{-(q+1) t-q}+1}{\prod_{t=0}^{6}\left(\Omega_{-(q+1) t-q} / \Omega_{-(3 q+2)}\right)}=\sum_{h=0}^{\infty} \prod_{k=1}^{7 h+4} \frac{1}{\prod_{t=0}^{5} \Omega_{(q+1) k-(q+1) t-q}+1} .
\end{aligned}
$$


Similarly,

$$
\begin{aligned}
\lim _{n \longrightarrow \infty} \Omega_{(7 q+7) n+5 q+6}= & \lim _{n \longrightarrow \infty} \Omega_{-(2 q+1)}\left(1-\frac{\prod_{t=0}^{6}\left(\Omega_{-(q+1) t-q} / \Omega_{-(2 q+1)}\right)}{\prod_{t=0}^{5} \Omega_{-(q+1) t-q}+1}\right. \\
& \left.\times \sum_{h=0}^{n} \prod_{k=1}^{7 h+5} \frac{1}{\prod_{t=0}^{5} \Omega_{-(q+1) k-(q+1) t-q}+1}\right), \\
a_{5 q+6}= & \Omega_{-(2 q+1)}\left(1-\frac{\prod_{t=0}^{6}\left(\Omega_{-(q+1) t-q} / \Omega_{-(2 q+1)}\right)}{\prod_{t=0}^{5} \Omega_{-(q+1) t-q}+1}\right. \\
& \left.\times \sum_{h=0}^{n} \prod_{k=1}^{7 h+5} \frac{1}{\prod_{t=0}^{5} \Omega_{-(q+1) k-(q+1) t-q}+1}\right), \\
a_{5 q+6}= & 0 \frac{\prod_{t=0}^{5} \Omega_{-(q+1) t-q}+1}{\prod_{t=0}^{6}\left(\Omega_{-(q+1) t-q} / \Omega_{-(2 q+1)}\right)}=\sum_{h=0}^{\infty} \prod_{k=1}^{7 h+5} \frac{1}{\prod_{t=0}^{5} \Omega_{(q+1) k-(q+1) t-q}+1} .
\end{aligned}
$$

Similarly,

$$
\begin{aligned}
\lim _{n \longrightarrow \infty} \Omega_{(7 q+7) n+6 q+7}= & \lim _{n \longrightarrow \infty} \Omega_{-(q)}\left(1-\frac{\prod_{t=0}^{6}\left(\Omega_{-(q+1) t-q} / \Omega_{-(2 q+1)}\right)}{\prod_{t=0}^{5} \Omega_{-(q+1) t-q}+1} \times\right. \\
& \left.\times \sum_{h=0}^{n} \prod_{k=1}^{7 h+6} \frac{1}{\prod_{t=0}^{5} \Omega_{-(q+1) k-(q+1) t-q}+1}\right), \\
a_{6 q+7}= & \Omega_{-(q)}\left(1-\frac{\prod_{t=0}^{6}\left(\Omega_{-(q+1) t-q} / \Omega_{-(q)}\right)}{\prod_{t=0}^{5} \Omega_{-(q+1) t-q}+1} \sum_{h=0}^{n} \prod_{k=1}^{7 h+6} \frac{1}{\prod_{t=0}^{5} \Omega_{-(q+1) k-(q+1) t-q}+1}\right), \\
a_{6 q+7}= & 0 \Rightarrow \frac{\prod_{t=0}^{5} \Omega_{-(q+1) t-q}+1}{\prod_{t=0}^{6}\left(\Omega_{-(q+1) t-q} / \Omega_{-(q)}\right)}=\sum_{h=0}^{\infty} \prod_{k=1}^{7 h+6} \frac{1}{\prod_{t=0}^{5} \Omega_{(q+1) k-(q+1) t-q}+1} .
\end{aligned}
$$

From equations (29) and (30),

$$
\begin{gathered}
\frac{\prod_{t=0}^{5} \Omega_{-(q+1) t-q}+1}{\prod_{t=0}^{5} \Omega_{-(q+1) t-q}}=\sum_{h=0}^{\infty} \prod_{k=1}^{7 h} \frac{1}{\prod_{t=0}^{5} \Omega_{(q+1) k-(q+1) t-q}+1}> \\
\frac{\prod_{t=0}^{5} \Omega_{-(q+1) t-q}+1}{\prod_{t=0}^{6}\left(\Omega_{-(q+1) t-q} / \Omega_{-(6 q+5)}\right)}=\sum_{h=0}^{\infty} \prod_{k=1}^{7 h+1} \frac{1}{\prod_{t=0}^{5} \Omega_{(q+1) k-(q+1) t-q}+1} .
\end{gathered}
$$


Thus, $\Omega_{-(7 q+6)}>\Omega_{-(6 q+5)}$.

From equations (30) and (31),

$$
\begin{aligned}
& \frac{\prod_{t=0}^{5} \Omega_{-(q+1) t-q}+1}{\prod_{t=0}^{6}\left(\Omega_{-(q+1) t-q} / \Omega_{-(6 q+5)}\right)}=\sum_{h=0}^{\infty} \prod_{k=1}^{7 h+1} \frac{1}{\prod_{t=0}^{5} \Omega_{(q+1) k-(q+1) t-q}+1}> \\
& \frac{\prod_{t=0}^{5} \Omega_{-(q+1) t-q}+1}{\prod_{t=0}^{6}\left(\Omega_{-(q+1) t-q} / \Omega_{-(5 q+4)}\right)}=\sum_{h=0}^{\infty} \prod_{k=1}^{7 h+2} \frac{1}{\prod_{t=0}^{5} \Omega_{(q+1) k-(q+1) t-q}+1} .
\end{aligned}
$$

Thus, $\Omega_{-(6 q+5)}>\Omega_{-(5 q+4)}$.

From equations (31) and (32),

$$
\begin{aligned}
& \frac{\prod_{t=0}^{5} \Omega_{-(q+1) t-q}+1}{\prod_{t=0}^{6}\left(\Omega_{-(q+1) t-q} / \Omega_{-(5 q+4)}\right)}=\sum_{h=0}^{\infty} \prod_{k=1}^{7 h+2} \frac{1}{\prod_{t=0}^{5} \Omega_{(q+1) k-(q+1) t-q}+1}> \\
& \frac{\prod_{t=0}^{5} \Omega_{-(q+1) t-q+1}+1}{\prod_{t=0}^{6}\left(\Omega_{-(q+1) t-q} / \Omega_{-(4 q+3)}\right)}=\sum_{h=0}^{\infty} \prod_{k=1}^{7 h+3} \frac{1}{\prod_{t=0}^{5} \Omega_{(q+1) k-(q+1) t-q}+1} .
\end{aligned}
$$

Thus, $\Omega_{-(5 q+4)}>\Omega_{-(4 q+3)}$.

From equations (32) and (33),

$$
\begin{aligned}
& \frac{\prod_{t=0}^{5} \Omega_{-(q+1) t-q}+1}{\prod_{t=0}^{6}\left(\Omega_{-(q+1) t-q} / \Omega_{-(4 q+3)}\right)}=\sum_{h=0}^{\infty} \prod_{k=1}^{7 h+3} \frac{1}{\prod_{t=0}^{5} \Omega_{(q+1) k-(q+1) t-q}+1}> \\
& \frac{\prod_{t=0}^{5} \Omega_{-(q+1) t-q}+1}{\prod_{t=0}^{6}\left(\Omega_{-(q+1) t-q} / \Omega_{-(3 q+2)}\right)}=\sum_{h=0}^{\infty} \prod_{k=1}^{7 h+4} \frac{1}{\prod_{t=0}^{5} \Omega_{(q+1) k-(q+1) t-q}+1} .
\end{aligned}
$$

Thus, $\Omega_{-(4 q+3)}>\Omega_{-(3 q+2)}$.

From equations (33) and (34),

$$
\begin{aligned}
& \frac{\prod_{t=0}^{5} \Omega_{-(q+1) t-q}+1}{\prod_{t=0}^{6}\left(\Omega_{-(q+1) t-q} / \Omega_{-(3 q+2)}\right)}=\sum_{h=0}^{\infty} \prod_{k=1}^{7 h+4} \frac{1}{\prod_{t=0}^{5} \Omega_{(q+1) k-(q+1) t-q}+1}> \\
& \frac{\prod_{t=0}^{5} \Omega_{-(q+1) t-q}+1}{\prod_{t=0}^{6}\left(\Omega_{-(q+1) t-q} / \Omega_{-(2 q+1)}\right)}=\sum_{h=0}^{\infty} \prod_{k=1}^{7 h+5} \frac{1}{\prod_{t=0}^{5} \Omega_{(q+1) k-(q+1) t-q}+1} .
\end{aligned}
$$

Thus, $\Omega_{-(3 q+2)}>\Omega_{-(2 q+1)}$.

From equations (34) and (35),

$$
\begin{gathered}
\frac{\prod_{t=0}^{5} \Omega_{-(q+1) t-q}+1}{\prod_{t=0}^{6}\left(\Omega_{-(q+1) t-q} / \Omega_{-(2 q+1)}\right)}=\sum_{h=0}^{\infty} \prod_{k=1}^{7 h+5} \frac{1}{\prod_{t=0}^{5} \Omega_{(q+1) k-(q+1) t-q+1}}> \\
\frac{\prod_{t=0}^{5} \Omega_{-(q+1) t-q}+1}{\prod_{t=0}^{6}\left(\Omega_{-(q+1) t-q} / \Omega_{-(q)}\right)}=\sum_{h=0}^{\infty} \prod_{k=1}^{7 h+6} \frac{1}{\prod_{t=0}^{5} \Omega_{(q+1) k-(q+1) t-q}+1} .
\end{gathered}
$$




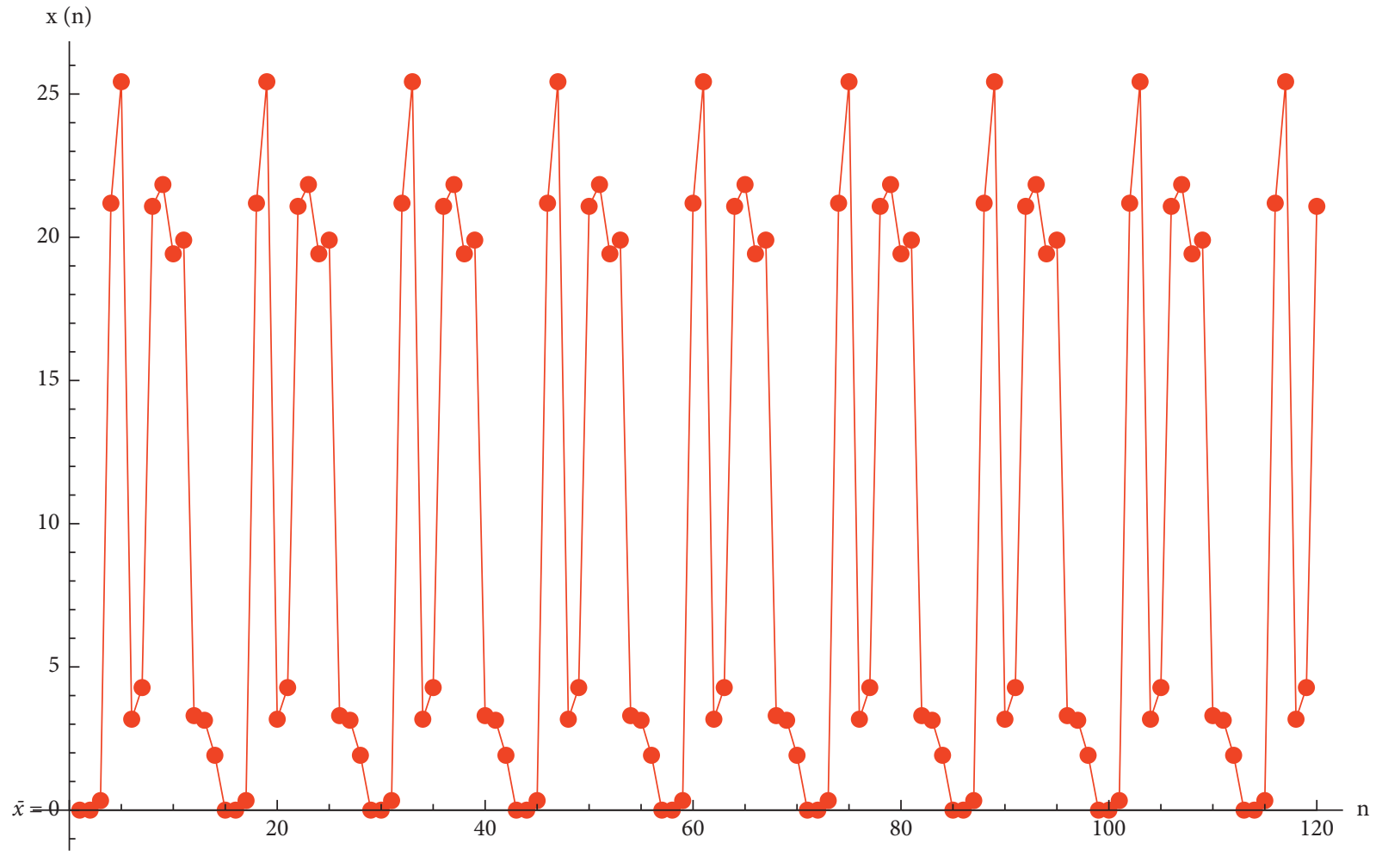

Figure 1: Dynamics of equation (5) with initial conditions in Example 1.

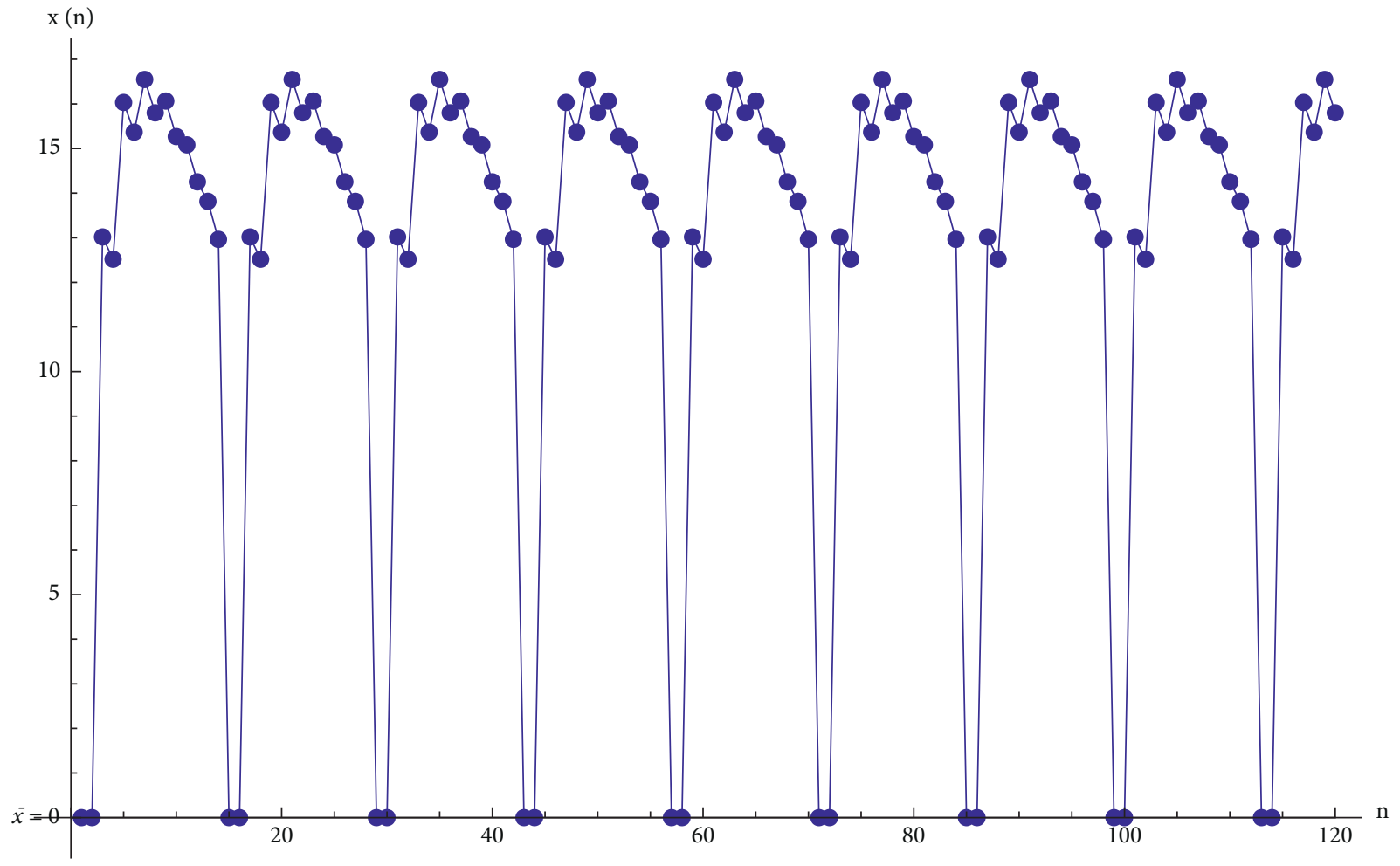

FIgUre 2: Dynamics of equation (5) with initial conditions in Example 2. 
Thus, $\Omega_{-(2 q+1)}>\Omega_{-(q)}$.

$$
\Omega_{-(7 q+6)}>\Omega_{-(6 q+5)}>\Omega_{-(5 q+4)}>\Omega_{-(4 q+3)}>\Omega_{-(3 q+2)}>\Omega_{-(2 q+1)}>\Omega_{-(q)} .
$$

\section{Examples}

In this section, we consider some numerical examples.

Example 1. Assume that, for $q=1$, we get $\Omega_{m+1}=\left(\Omega_{-13} /\left(1+\Omega_{-1} \Omega_{-3} \Omega_{-5} \Omega_{-7} \Omega_{-9} \Omega_{-11}\right)\right)$.

$$
\begin{aligned}
& \Omega_{-13}=10, \\
& \Omega_{-12}=9, \\
& \Omega_{-11}=2, \\
& \Omega_{-10}=28, \\
& \Omega_{-9}=27, \\
& \Omega_{-8}=6.5, \\
& \Omega_{-7}=5.5, \\
& \Omega_{-6}=24, \\
& \Omega_{-5}=23, \\
& \Omega_{-4}=22, \\
& \Omega_{-3}=21, \\
& \Omega_{-2}=5, \\
& \Omega_{-1}=4, \\
& \Omega_{0}=3 .
\end{aligned}
$$

Then, we have the graph in Figure 1.

Example 2. If we select the initial conditions as follows,

$$
\begin{aligned}
& \Omega_{-13}=29, \\
& \Omega_{-12}=28, \\
& \Omega_{-11}=27, \\
& \Omega_{-10}=26, \\
& \Omega_{-9}=25, \\
& \Omega_{-8}=24, \\
& \Omega_{-7}=23, \\
& \Omega_{-6}=22, \\
& \Omega_{-5}=21, \\
& \Omega_{-4}=20, \\
& \Omega_{-3}=19, \\
& \Omega_{-2}=18, \\
& \Omega_{-1}=17, \\
& \Omega_{0}=16,
\end{aligned}
$$

then we have the graph in Figure 2.

\section{Data Availability}

All the data utilized in this article have been included, and the sources from where they were adopted are cited accordingly.

\section{Conflicts of Interest}

The authors declare that they have no conflicts of interest.

\section{References}

[1] C. Cinar, "On the positive solutions of the difference equation $\Omega_{m+1}=\left(\Omega_{m-1} /\left(1+\alpha \Omega_{m} \Omega_{m-1}\right)\right)$," International Journal of Applied Mathematics and Computer Science, vol. 158, pp. 890-812, 2004

[2] C. Cinar, "On the solutions of the difference equation $\Omega_{m+1}=\left(\Omega_{m-1} /\left(-1+\alpha \Omega_{m} \Omega_{m-1}\right)\right)$," Applied Mathematics and Computation, vol. 158, no. 3, pp. 793-797, 2004.

[3] C. Çinar, "On the positive solutions of the difference equation $\Omega_{m+1}=\left(\alpha \Omega_{m-1} /\left(1+b \Omega_{m} \Omega_{m-1}\right)\right)$," Applied Mathematics and Computation, vol. 156, no. 2, pp. 587-590, 2004.

[4] R. DeVault, G. Ladas, and S. Schultz, "On the recursive sequence $\Omega_{m+1}=\left(A / \Omega_{m}\right)+\left(1 / \Omega_{m-2}\right)$," Proceedings of the American Mathematical Society, vol. 126, no. 11, pp. 32573261, 1998.

[5] E. M. Elsayed, "Dynamics and behavior of a higher order rational difference equation," The Journal of Nonlinear Science and Applications, vol. 9, no. 4, pp. 1463-1474, 2016.

[6] D. Simsek, C. Cinar, and I. Yalcinkaya, "On the recursive sequence $\Omega_{m+1}=\left(\Omega_{m-3} /\left(1+\Omega_{m-1}\right)\right)$," International Journal of Contemporary Mathematical Sciences, vol. 9, no. 12, pp. 475-480, 2006.

[7] D. Simsek, C. Cinar, and I. Yalcinkaya, "On the recursive sequence $\Omega_{m+1}=\left(\Omega_{m-5} /\left(1+\Omega_{m-2}\right)\right)$," International Journal of Pure and Applied Mathematics, vol. 27, pp. 501-507, 2006.

[8] D. Simsek, C. Cinar, and I. Yalcinkaya, "On the recursive sequence $\Omega_{m+1}=\left(\Omega_{m-5} /\left(1+\Omega_{m-1} \Omega_{m-3}\right)\right)$," International Journal of Pure and Applied Mathematics, vol. 28, pp. 117-124, 2006.

[9] D. Simsek and M. Eroz, "Solutions of the rational difference equations $\Omega_{m+1}=\left(\Omega_{m-3} /\left(1+\Omega_{m} \Omega_{m-1} \Omega_{m-2}\right)\right)$," Manas Journal of Engineering, vol. 4, no. 1, pp. 12-20, 2016.

[10] R. P. Agarwal, E. M. Elsayed, On the solution of fourth-order rational recursive sequence," Advanced Studies in Contemporary Mathematics, vol. 20, no. 4, pp. 525-545, 2010.

[11] A. M. Amleh, E. A. Grove, G. Ladas, and D. A. Georgiou, "On the recursive sequence $y_{n+1}=\alpha+\left(y_{n-1} / y_{n}\right)$," Journal of Mathematical Analysis and Applications, vol. 233, no. 2, pp. 790-798, 1999.

[12] C. Cinar, T. Mansour, and I. Yalcinkaya, "On the difference equation of higher order," Utilitas Mathematica, vol. 92, pp. 161-166, 2013.

[13] S. E. Das and M. Bayram, "On a system of rational difference equations," World Applied Sciences Journal, vol. 10, no. 11, pp. 1306-1312, 2010. 
[14] M. E. Erdogan, C. Cinar, and I. Yalcinkaya, "On the dynamics of the recursive sequence," Mathematical and Computer Modelling, vol. 54, no. 5, pp. 1481-1485, 2011.

[15] C. H. Gibbons, M. R. S. Kulenovic, and G. Ladas, "On the recursive sequence $\left(\alpha+\beta x_{n-1}\right) /\left(\chi+\beta x_{n-1}\right)$," Research in the Mathematical Sciences, vol. 4, no. 2, pp. 1-11, 2000.

[16] T. F. Ibrahim, "Generalized partial todd's difference equation in $n$-dimensional space," Journal of Computational Analysis and Applications, vol. 26, no. 5, pp. 910-926, 2019.

[17] R. Karatas, C. Cinar, and D. Simsek, "On positive solutions of the difference equation $\Omega_{m+1}=\left(\Omega_{m-5} /\left(1+\Omega_{m-2} \Omega_{m-5}\right)\right)$," International Journal of Contemporary Mathematical Sciences, vol. 10, no. 1, pp. 495-500, 2006.

[18] A. Q. Khan and T. F. Ibrahim, "Stability and bifurcations analysis of discrete dynamical systems," Discrete Dynamics in Nature and Society, vol. 2019, Article ID 8474706, 2 pages, 2019.

[19] M. R. S. Kulenovic, G. Ladas, and W. S. Sizer, "On the recursive sequence $\left(\alpha \Omega_{m}+\beta \Omega_{m-1}\right) /\left(\chi \Omega_{m}+\beta \Omega_{m-1}\right)$," Research in the Mathematical Sciences, vol. 2, no. 5, pp. 1-16, 1998.

[20] M. Lui and Z. Guo, "Solvability of a higher-order nonlinear neutral delay difference equation," Advances in Difference Equations, vol. 2010, no. 1, pp. 620-627, Article ID 767620, 2010.

[21] H. D. Voulov, "Periodic solutions to a difference equation with maximum," Proceedings of the American Mathematical Society, vol. 131, no. 7, pp. 2155-2160, 2002. 